\title{
Performance analysis comparison between non mixed-criticality and mixed-criticality system in microcontroller
}

\author{
Siti Nurhafizza Maidin ${ }^{1}$, Noor Azurati Ahmad ${ }^{2}$, Kamilia Kamardin ${ }^{3}$, Shamsul Sahibuddin ${ }^{4}$, \\ Syahrizal Fadhlie Sabri ${ }^{5}$ \\ 1,2,4,5AIS department, Razak Faculty of Informatics and Technology, Universiti Teknologi Malaysia, Malaysia \\ ${ }^{3}$ Malaysian-Japanese International Institute of Technology, Universiti Teknologi Malaysia, Malaysia
}

\begin{tabular}{l}
\hline Article Info \\
\hline Article history: \\
Received Oct 15, 2018 \\
Revised Jan 18, 2019 \\
Accepted Mar 12, 2019 \\
\hline
\end{tabular}

Keywords:

Energy-saving

Dynamic power management (DPM)

Microcontroller

Mixed-criticality system

LabVIEW

\begin{abstract}
Nowadays, implementation of real-time embedded system or safety-critical in a real-time system is significant within emerging technologies because the system involves many aspects such as safety and task execution without missing deadlines. The main cause of implementation is to avoid catastrophic loss. Besides that, effectuation of the mixed-criticality system in embedded system making system more complex for task execution. For an embedded system, the main component involves real-time scheduling. The implementation of DPM method in real-time scheduling is well known, but in the mixed-criticality system, DPM method is still lacking. In order to cater this problem, Dynamic Power management (DPM) method is deployed onto the microcontroller of the mixed-criticality system to save energy when executing tasks in order to have better performance in the system. The usage of the DPM method in mixed-criticality of microcontroller resulting decrease of $0.82 \%$ in LED output voltage value meanwhile, for the LCD output, the voltage value decreased by $1.37 \%$ in the home alarm system. Thus, the energy-saving in the microcontroller of the mixed-criticality system using the DPM method is defined.
\end{abstract}

Copyright $@ 2019$ Institute of Advanced Engineering and Science. All rights reserved.

\section{Corresponding Author:}

Noor Azurati Ahmad,

AIS department, Razak Faculty of Informatics and Technology,

Universiti Teknologi Malaysia,

Kuala Lumpur, Malaysia.

Email: azurati@utm.my

\section{INTRODUCTION}

Microcontroller is widely used in a real-time system due to it's of performing heavy-duty or complex task during execution. However, when the microcontroller is executing tasks, high energy usage or energy leakage to surrounding could occur because of the powering up of unnecessary components. For the mixed-criticality system, microcontroller needs to have better instruction or an RTOS in order to execute task quickly. Besides that, the size of the microcontroller is decreasing and smaller nowadays and a chip mounted on microcontroller resulting in smarter execution of the microcontroller. The chip nowadays can multitasks such as by monitoring and controlling connected farm. Thus, many microcontroller manufacturers are now competing to develop microcontroller that can perform and show better performance with the benefits of high performance but low power or energy consumption in the system [1].

Moreover, mixed-criticality systems require significant challenges to design safety-critical real-time embedded systems [2, 3]. In the mixed-criticality system, safety-critical is one of the important characteristics because of priority task scheduling. Figure 1 shows an example of a safety-critical system for a vehicle. In the safety-critical system, high-priority task commonly preempts low-priority task at a certain level to ensure quickest response making safety as the first priority. In addition, in a safety-critical system, they define some tasks into either low-priority or high-priority because of safety element involvement [4]. 
Besides, the requirement placed upon safety-critical computer system increased significantly in size and complexity, and continued to increase at a very rapid place [5]. Furthermore, the higher the criticality level, the greater is the need for the task to complete well before any safety-critical timing constraint and hence the smaller the value of the relative deadline parameter [6]. However, in a complex system, the declaration of high-priority task is commonly used to avoid missed deadline and severe damage, meanwhile for low-priority task, missed deadline in the system does not affect system much. In many applications, the consequence of missing dateline vary in severity from task to task [7].

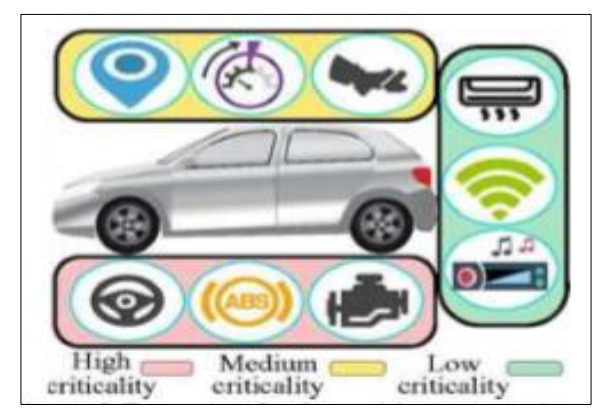

Figure 1. Example of safety-critical system in car

During task execution in the mixed-criticality system, voltage and frequency used to perform a task is usually high and is not efficiently preserved as in mixed-criticality system, a task needs to be executed fast. Besides that, utilization of frequency and voltage in the mixed-criticality system for executing varieties of task scheduled is not expeditiously handled, resulting in severe energy loss or energy leakage to the surroundings. In addition, with shared resources in the microcontroller, limitation of memory can happen, thus it will lead to insufficient energy to perform tasks. In the mixed-criticality system, shared resources fetched needs to be accurate and smooth in order to avoid any possible mistakes during task execution. Thus, the power consumption in the mixed-criticality system needs to be efficaciously managed to preserve energy. Manipulation of voltage in the mixed-criticality system helps to conserve more energy.

The introduction of mixed-criticality system scheduler is one of the ways to preserve energy during task execution. Implementation of DPM method in the microcontroller for the mixed-criticality system is significant as it is energy saving and has low power consumption. By having an effective method in the system, execution of the high-priority task can be done easily and energy saving could occur. Thus, it shows the significant function of the energy-saving method in the mixed-criticality system on the microcontroller.

Therefore, this project is aimed to refine the method of DPM in the microcontroller for the mixedcriticality system. This paper will investigate how DPM method handles the task in the mixed-criticality system by shutting down unnecessary components as the components can be put into idle state by disabling the clock [8]. This project is done using microcontroller provided to perceive voltage used in executing task and analyses the voltage value when task execution uses LabVIEW and NI-DAQ device. Section 2 of this article discusses the existing work related to the DPM method. The flowchart, case study system, and experiment are described in Section 3. Subsequent section will discuss the result of the experiment. Finally, Section 5 concludes this project.

\section{RELATED WORK}

Despite being a complex system, the implementation of mixed-criticality system in microcontroller or microprocessor is an emerging trends nowadays. A current trend in real-time embedded systems, driven by size, weight, power and cost concerns, is merging in many complex software tasks [9]. In order to perform integrated multiple functions or tasks with a different degree of importance or criticalities, the deployment of task in common platform or integrated platform [10] is needed to ensure the execution of the task is successful. However, power consumption has been an overriding concern in the design of computers [11] system and the power consumption in the real-time embedded systems has become an important issue. It is because processors consume a large portion of energy around 50\% of the overall consumed energy of computer [12,13]. Some scheduler or methods had been introduced to save the energy in the microcontroller.

The new version of FlexPRET processor is focusing on the mixed-criticality system [9]. It uses the fine-grained multithreaded processor, which is designed to exhibit architectural techniques, useful for the 
mixed-criticality system. The scheduling used in the FlexPRET is based on round robin scheduling where the execution of the task is without priority. The thread scheduler used in this processor is based on two control registers that are slots control register and thread mode control register. The processor will schedule the task based on this two control registers.

Another scheduler introduced is dynamic power-aware scheduling which is implemented in the mixed-criticality systems. The scheme used in the scheduling is to make use of the slack time by reducing the energy in the mixed-criticality for high-priority reservation [2]. The algorithm used in the scheduling is Earliest Deadline First-Virtual Deadline (EDF-VD) that can provide an optimal virtual deadline and frequency levels of tasks. Thus, it can decrease the power consumption in the system as the frequency is dynamically being adjusted.

The global mixed-criticality scheduling is introduced where fpEDF implementation is used in the task scheduling [10]. The deployment of three phases in the fpEDF algorithm is pre-processing, runtime phase and an execution phase. It results in the schedulable mixed-criticality (MC) implicit-deadline sporadic task system for pre-processing phase and whenever some job is executing more than the LO-criticality WCET without sending a signal of completion. The introduction of algorithm GLOBAL and GLOBALPRAGMATIC is done when the values of $x$ is the value for scaling factor cannot be found in the fpEDF algorithm.

The new algorithm, Priority List Reuse Scheduling (PLRS) is used to solve the problems arising in the LB [14] which are the performance of LB and the large run-time overhead of LB. The deployment of offline computation and the run-time scheduling is the technique for PLRS to solve the problems arising in LB. PLRS not only theoretically dominates, but also on average, it significantly outperforms LB in terms of acceptance ratios and also the run-time complexity of PLRS is polynomial (quadratic in the number of tasks), thereby is much more efficient than the pseudo-polynomial run-time scheduling of LB. In practice, PLRS's run-time overhead can be several orders of magnitude smaller than LB's.

In [15], usage of the DPM and DVFS algorithm for energy saving in the microcontroller is done. The function of the algorithms is to vary the voltage of each microcontroller involved, varies the frequency based on the system workload and other I/O devices and selectively switches off the system when in idle mode. The algorithm used focuses on the embedded system in the microcontroller.

Meanwhile, in [16], implementation of DPM and DVFS method in the microcontroller for embedded system is done and results in $47 \%$ energy-saving for sole use and $12 \%$ energy-saving on the core for the whole microcontroller. The algorithm function is to supply different voltages applied to each processor, shut off one processor to switch to a single processor configuration, varies the component voltage and selectively turns off the system whenever microcontroller is in the idle mode.

\section{METHODOLOGY}

\subsection{Block Diagram of System \& Flowchart of Mixed-Criticality System}

Figure 2 shows block diagram of system used to conduct this project. There are 3 elements that are very important in this project. Elements involved are microcontroller (which consists of mixed-criticality system), DPM method (the triggered factor in the system to preserve energy) and low energy consumption (the main output for the system). These 3 elements show the importance of the project is conducted in order to preserve energy in mixed-criticality (MC) system using DPM method.



Figure 2. Block diagram of the system

Figure 3 shows flowchart of mixed-criticality in home alarm system. The flowchart begins with start and then goes to input is task assignation priority and input data from the potentiometer. After that, the process to assign the task to priority mode is done where the first task is assigned as low-criticality mode, 
task two can be assigned as low or high-criticality mode and third task are assigned as high-criticality mode. Then, the condition is done based on the input data from the potentiometer. When the temperature detected is less or equal to 36 degrees, the first task is assigned as low-criticality mode, task two is assigned as low mode and third task is assigned as high-criticality mode for output of the process. However, if the temperature detected is more than or equal to 38 degrees, the first task is assigned as low-criticality mode, task two is assigned as high-criticality mode and third task is assigned as high-criticality mode priority is assign as lowcriticality mode as output of the process. If there is no temperature detected, it will go to the end. Temperature of 36 degrees and 38 degrees is the threshold that has been used in the home alarm system case study. This is to ignite the given priority task involved in the home alarm system.

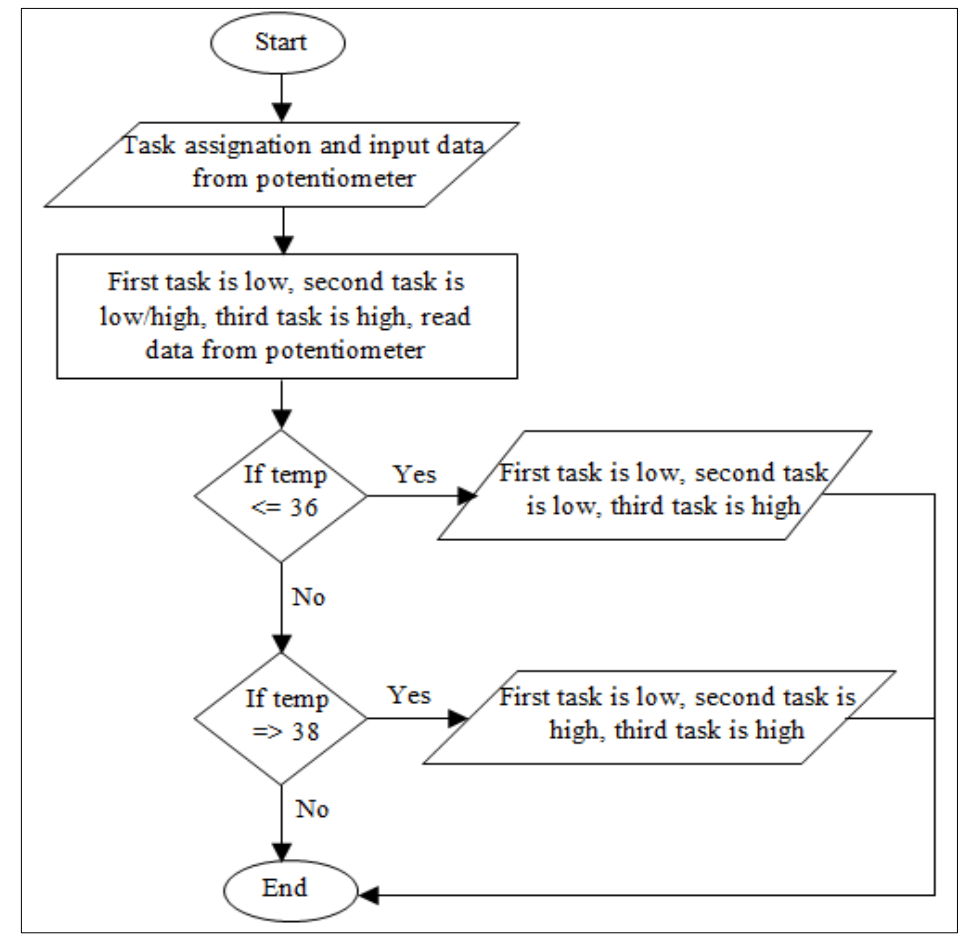

Figure 3. Flowchart of mixed-criticality in home alarm system

\subsection{Case study (Home alarm system)}

In order to see the effectiveness of DPM method in terms of energy saving in the microcontroller (contain the mixed-criticality system), the home alarm system is used as the case study and act as the realtime embedded system with the mixed-criticality system. The home alarm system is used to monitor temperature in the house and alert residence of the house about increasing temperature in the house. In home alarm system, tasks are defined as task of LCD, LED and loudspeaker. The LCD task has highest priority task which is task 2 and task loudspeaker has second highest priority task which is 1 meanwhile lowest priority task is given to LED which is 0 . The task of LCD, LED and loudspeaker are the output of the home alarm system, meanwhile the input for the tasks is taken from the potentiometer that acts as temperature sensor in the home alarm system.

Figure 4 shows how the potentiometer will act as the temperature sensor to give input to the home alarm system. The reading of potentiometer starts from 0x0000 to 0x03FF as shown in Figure 5. For each phase of potentiometer, it indicates the temperature involved for the home alarm system. In the first condition or the first phase of potentiometer reading, if the potentiometer reading is less than 0x0065 (or temperature is equal or less than 32 degrees), it will turn on LED 1. If potentiometer reading is equal to 0x0065 and less than $0 \mathrm{x} 021 \mathrm{~F}$ (or temperature is equal or less than 34 degrees), LED 2 will turn on for condition 2 or second phase of potentiometer reading. In condition 3 or the third phase of potentiometer reading, if potentiometer reading is equal to $0 \mathrm{x} 021 \mathrm{~F}$ and less than $0 \mathrm{x} 03 \mathrm{C} 9$ (or temperature is equal or less 36 degree), LED 3 will turn on. In condition 4 or last phase of potentiometer reading, LED 4 turn idle, the LCD shows a message and loudspeaker turn on if the potentiometer reading is equal and higher than $0 \mathrm{x} 03 \mathrm{C} 9$ (or temperature is equal or 
more than 38 degrees). The LEDs, LCD and loudspeaker will function based on the range of the potentiometer reading as the input data.

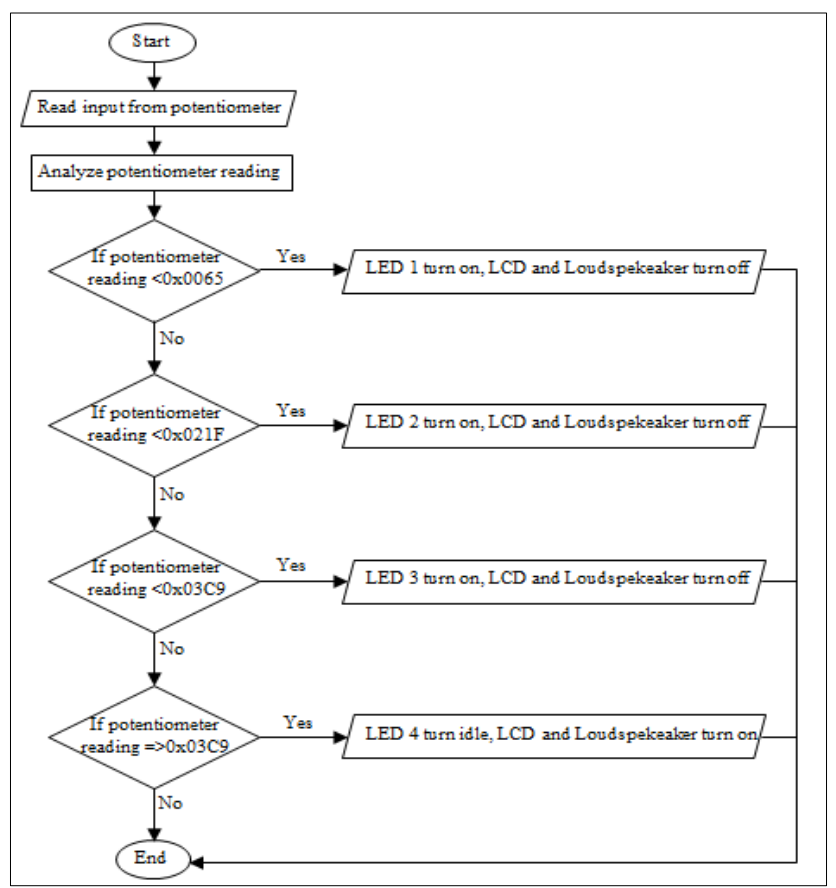

Figure 4. Flowchart of case study

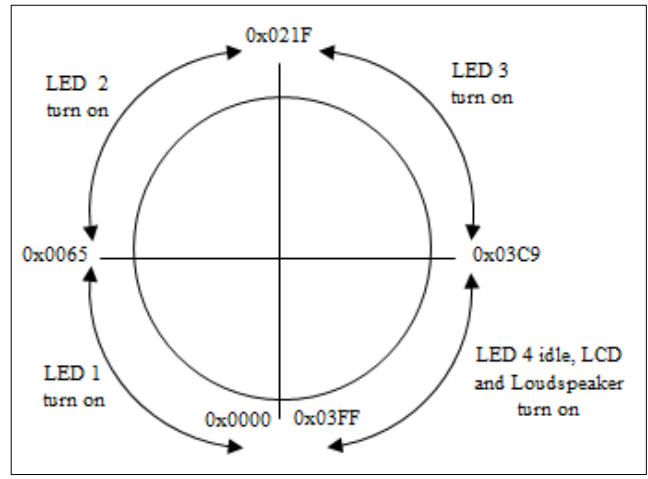

Figure 5. Potentiometer reading range of home alarm system

\subsection{Simulation \& Experiment}

The experiment was done using a simulator called LabVIEW. LabVIEW is used to develop block diagram of voltage reading for case study system. The connection between microcontroller (MCB2300) and NI-DAQ using jumpers and header is also done to measure voltage and energy usage during task execution and no task execution in phase 3 and phase 4 . Each of the output is connected to one channel and voltage value is measured during task execution and no task execution. Better understanding of the result obtained can be done when the result is being written in excel, so that analysis on the result can be done. Besides that, the software to download the home alarm system into microcontroller is called Keil uVision 5. Keil uVision 5 is used for home alarm system programming and LabVIEW, which is used to design connection between NI-DAQ and voltage channel to measure voltage when task is executed. After the coding for the home alarm system is done in the Keil uVision 5, the code is downloaded into the microcontroller in order to test the code that consists of elements of mixed-criticality.

\section{RESULTS OF EXPERIMENT}

The implementation phase will focus on case study implementation of home alarm system that acts as real-time embedded system with mixed criticality system. The experiment is used to see the effectiveness of DPM method done on non-mixed-criticality system and mixed-criticality system. Differences between non-mixed-criticality system and mixed-criticality system is the priority set up. For mixed-criticality system, the priority is being set up meanwhile for the non-mixed-criticality system, no priority is being set up. For mixed-criticality system, priority being set up for each task is significant because it can help the system to meet deadlines and avoid any catastrophic effect from missed deadlines.

\subsection{Performance Analysis}

\subsubsection{Phase 3}

A. Non-Mixed-Criticality System

In this section, results of the non-mixed-criticality system (non-MC) on the voltage calculated is discussed and the result is stored in the excel format. For each of the result, the voltage is calculated when the task executes or no task is executed using the NI-DAQ device. The result obtained using the NI-DAQ is very 
useful because the result then can be analyzed and can help to see how the DPM method can benefit the mixed-criticality system.

Table 1 shows the voltage calculated using the NI-DAQ device when the device is connected to each of the outputs. The value of 0.001 until 0.01 shows number of samples taken from the result and overall number of samples taken is 1250 samples. For this project, only 10 samples are taken to show voltage difference in each phase. It shows that the voltage calculated in the task executed is lower compared to the no task executes. Phase 3 focuses on the LED because in the phase 3, the LED 3 will turn on when the potentiometer reading is in range of 0x0220 until 0x03C9. Increase in number of samples decreases the value of voltage in the LED.

Table 1. Result of the Voltage Measured using NI-DAQ Device in Phase 3 for Non-MC System

\begin{tabular}{|c|c|c|c|}
\hline \multicolumn{4}{|c|}{ No task execute in non MC system } \\
\hline & \multicolumn{3}{|c|}{ Voltage measured (V) } \\
\hline No. of samples & LED & LCD & Loudspeaker \\
\hline 0 & 3.181894 & 1.543914 & 2.416386 \\
\hline 0.001 & 3.233443 & 2.33133 & 2.380302 \\
\hline 0.002 & 3.221844 & 2.680576 & 2.485978 \\
\hline 0.003 & 3.211534 & 2.839091 & 2.60583 \\
\hline 0.004 & 3.211534 & 2.917703 & 2.702485 \\
\hline 0.005 & 3.205091 & 2.956365 & 2.770788 \\
\hline 0.006 & 3.202513 & 2.980851 & 2.823626 \\
\hline 0.007 & 3.203802 & 2.998894 & 2.85971 \\
\hline 0.008 & 3.202513 & 3.01307 & 2.889351 \\
\hline 0.009 & 3.199936 & 3.027246 & 2.920281 \\
\hline 0.01 & 3.197358 & 3.040133 & 2.944767 \\
\hline
\end{tabular}

\begin{tabular}{|c|c|c|c|}
\hline \multicolumn{4}{|c|}{ Task execute in non MC system } \\
\hline & \multicolumn{3}{|c|}{ Voltage measured (V) } \\
\hline No. of samples & LED & LCD & Loudspeaker \\
\hline 0 & 3.18576 & 1.545202 & 2.424118 \\
\hline 0.001 & 3.23602 & 2.349372 & 2.397055 \\
\hline 0.002 & 3.216689 & 2.701196 & 2.52464 \\
\hline 0.003 & 3.203802 & 2.862288 & 2.654802 \\
\hline 0.004 & 3.193492 & 2.969253 & 2.779809 \\
\hline 0.005 & 3.192203 & 2.969253 & 2.842957 \\
\hline 0.006 & 3.184471 & 2.976985 & 2.835224 \\
\hline 0.007 & 3.190915 & 2.962809 & 2.860999 \\
\hline 0.008 & 3.199936 & 3.036267 & 2.876464 \\
\hline 0.009 & 3.198647 & 2.980851 & 2.882908 \\
\hline 0.01 & 3.197358 & 3.019513 & 2.895795 \\
\hline
\end{tabular}

\section{B. Mixed-Criticality System}

In this section, the result of mixed-criticality system (MC) on the voltage is calculated and the result is stored in excel format. Based on the excel file, the performance analysis is done to see how the performance of the system without DPM implementation.

Table 2 shows the result of voltage calculated using the NI-DAQ device when the device is connected to each of the outputs. It shows that the voltage calculated in the task execute is higher compared to the no task executes because in the no task execution, no output is functioning meanwhile in the task execution, the LED will function to give an output of the system. However, as the numbers of samples in the task execute increases, the voltage value of the LED decreases.

Table 2. Result of the Voltage Measured using NI-DAQ Device in Phase 3 for MC System

\begin{tabular}{|c|c|c|c|}
\hline \multicolumn{4}{|c|}{ No task execute in MC system } \\
\hline & \multicolumn{3}{|c|}{ Voltage measured (V) } \\
\hline No. of samples & LED & LCD & Loudspeaker \\
\hline 0 & 3.171584 & 1.556801 & 2.411231 \\
\hline 0.001 & 3.229577 & 2.368703 & 2.380302 \\
\hline 0.002 & 3.217978 & 2.720527 & 2.510464 \\
\hline 0.003 & 3.205091 & 2.88033 & 2.652224 \\
\hline 0.004 & 3.193492 & 2.953788 & 2.761767 \\
\hline 0.005 & 3.188337 & 2.989872 & 2.837802 \\
\hline 0.006 & 3.18576 & 3.01307 & 2.893217 \\
\hline 0.007 & 3.181894 & 3.024668 & 2.926724 \\
\hline 0.008 & 3.17545 & 3.029823 & 2.947344 \\
\hline 0.009 & 3.172872 & 3.036267 & 2.965386 \\
\hline 0.01 & 3.172872 & 3.040133 & 2.974408 \\
\hline
\end{tabular}

\begin{tabular}{|c|c|c|c|}
\hline \multicolumn{4}{|c|}{ Task execute in MC system } \\
\hline & \multicolumn{3}{|c|}{ Voltage measured (V) } \\
\hline No. of samples & LED & LCD & Loudspeaker \\
\hline 0 & 3.192203 & 1.541336 & 2.43314 \\
\hline 0.001 & 3.245042 & 2.362259 & 2.404788 \\
\hline 0.002 & 3.225711 & 2.721816 & 2.541393 \\
\hline 0.003 & 3.205091 & 2.884196 & 2.683154 \\
\hline 0.004 & 3.194781 & 2.953788 & 2.786253 \\
\hline 0.005 & 3.18576 & 2.984717 & 2.846823 \\
\hline 0.006 & 3.181894 & 2.998894 & 2.88033 \\
\hline 0.007 & 3.176739 & 3.000182 & 2.898372 \\
\hline 0.008 & 3.176739 & 2.997605 & 2.899661 \\
\hline 0.009 & 3.178027 & 3.06333 & 2.960232 \\
\hline 0.01 & 3.176739 & 3.028534 & 2.931879 \\
\hline
\end{tabular}

\subsubsection{Phase 4}

\section{A. Non-Mixed-Criticality System}

In this section, the result of non-mixed-criticality system on the voltage is calculated and the result is stored in excel format. Table 3 shows the result of the voltage calculated using the NI-DAQ device when the device is connected to each of the outputs of phase 4. It shows that the voltage calculated in the task execute is lower compared to the no task executes and the voltage value for each of the output is smaller for the task execution. 
Table 3. Result of the Voltage Measured using NI-DAQ Device in Phase 4 for Non-MC System

\begin{tabular}{|c|c|c|c|}
\hline \multicolumn{4}{|c|}{ No task execute in non MC system } \\
\hline & \multicolumn{3}{|c|}{ Voltage measured (V) } \\
\hline No. of samples & LED & LCD & Loudspeaker \\
\hline 0 & 3.198647 & 1.569688 & 0.853153 \\
\hline 0.001 & 3.221844 & 2.420252 & 1.663766 \\
\hline 0.002 & 3.187049 & 2.779809 & 2.21921 \\
\hline 0.003 & 3.167718 & 2.925436 & 2.547837 \\
\hline 0.004 & 3.157408 & 2.984717 & 2.730837 \\
\hline 0.005 & 3.148387 & 3.009203 & 2.832647 \\
\hline 0.006 & 3.147098 & 3.018225 & 2.884196 \\
\hline 0.007 & 3.148387 & 3.023379 & 2.908682 \\
\hline 0.008 & 3.147098 & 3.028534 & 2.930591 \\
\hline 0.009 & 3.150964 & 3.031112 & 2.940901 \\
\hline 0.01 & 3.148387 & 3.031112 & 2.947344 \\
\hline
\end{tabular}

\begin{tabular}{|c|c|c|c|}
\hline \multicolumn{4}{|c|}{ Task execute in non MC system } \\
\hline & \multicolumn{3}{|c|}{ Voltage measured (V) } \\
\hline No. of samples & LED & LCD & Loudspeaker \\
\hline 0 & 3.19607 & 1.518139 & 0.824801 \\
\hline 0.001 & 3.224422 & 2.389323 & 1.636703 \\
\hline 0.002 & 3.188337 & 2.756612 & 2.192147 \\
\hline 0.003 & 3.163851 & 2.907393 & 2.525928 \\
\hline 0.004 & 3.152253 & 2.969253 & 2.711506 \\
\hline 0.005 & 3.147098 & 2.995027 & 2.813316 \\
\hline 0.006 & 3.145809 & 3.006626 & 2.868731 \\
\hline 0.007 & 3.14452 & 3.011781 & 2.899661 \\
\hline 0.008 & 3.14452 & 3.014358 & 2.917703 \\
\hline 0.009 & 3.143232 & 3.019513 & 2.929302 \\
\hline 0.01 & 3.148387 & 3.020802 & 2.935746 \\
\hline
\end{tabular}

\section{B. Mixed-Criticality System}

In this section, the result of the mixed-criticality system on the voltage is calculated and the result is stored in excel format. In order to analyze the voltage value obtained using the NI-DAQ device, the voltage value in excel format can help analyze better. Based on the excel file, the performance analysis is done to see how the performance of the system without DPM implementation.

Table 4 shows the result of voltage calculated using the NI-DAQ device when the device is connected to each of the outputs. It shows that voltage calculated in the task execute is lower compared to the no task executes for each of the outputs in the home alarm system.

Table 4. Result of the Voltage Measured using NI-DAQ Device in Phase 4 for MC System

\begin{tabular}{|c|c|c|c|}
\hline \multicolumn{4}{|c|}{ No task execute in MC system } \\
\hline & \multicolumn{3}{|c|}{ Voltage measured (V) } \\
\hline No. of samples & LED & LCD & Loudspeaker \\
\hline 0 & 3.181894 & 1.503963 & 0.788716 \\
\hline 0.001 & 3.210246 & 2.353238 & 1.599329 \\
\hline 0.002 & 3.181894 & 2.716661 & 2.154773 \\
\hline 0.003 & 3.152253 & 2.871309 & 2.491133 \\
\hline 0.004 & 3.141943 & 2.938323 & 2.677999 \\
\hline 0.005 & 3.136788 & 2.969253 & 2.783675 \\
\hline 0.006 & 3.131633 & 2.98214 & 2.842957 \\
\hline 0.007 & 3.129056 & 2.988584 & 2.875175 \\
\hline 0.008 & 3.129056 & 2.989872 & 2.89064 \\
\hline 0.009 & 3.129056 & 2.99245 & 2.899661 \\
\hline 0.01 & 3.13421 & 2.995027 & 2.906105 \\
\hline
\end{tabular}

\begin{tabular}{|c|c|c|c|}
\hline \multicolumn{4}{|c|}{ Task execute in MC system } \\
\hline & \multicolumn{3}{|c|}{ Voltage measured (V) } \\
\hline No. of samples & LED & LCD & Loudspeaker \\
\hline 0 & 3.184471 & 1.509118 & 0.797737 \\
\hline 0.001 & 3.207668 & 2.357104 & 1.598041 \\
\hline 0.002 & 3.174161 & 2.719238 & 2.152196 \\
\hline 0.003 & 3.148387 & 2.867443 & 2.476957 \\
\hline 0.004 & 3.135499 & 2.930591 & 2.665112 \\
\hline 0.005 & 3.129056 & 2.96152 & 2.769499 \\
\hline 0.006 & 3.126478 & 2.974408 & 2.827492 \\
\hline 0.007 & 3.122612 & 2.980851 & 2.860999 \\
\hline 0.008 & 3.118746 & 2.984717 & 2.879041 \\
\hline 0.009 & 3.122612 & 2.986006 & 2.89064 \\
\hline 0.01 & 3.126478 & 2.989872 & 2.898372 \\
\hline
\end{tabular}

\subsubsection{Phase 3 VS Phase 4}

In this section, analysis of the result of phase 3 and phase 4 is done to see how the LED becomes idle in phase 4 meanwhile in phase 3, the LED is not idle. DPM method implementation of phase 4 is significant because the voltage value obtained using NI-DAQ device is lower with increased number of samples. Table 5 shows the difference in voltage value for phase 3 and phase 4.

Based on Table 5, the difference in voltage value measured for phase 3 and phase 4 can be seen where the voltage value for LED in phase 4 is high at first, but slowly decreases because of the DPM implementation meanwhile voltage value in phase 3 increased along with the increased number of samples. Similarly, the LCD output in phase 4 has higher priority compared to LED output, resulting LED to turn off selectively via DPM method. Based on Table 5, we can see the importance of DPM implementation in the mixed-criticality system. Average voltage for Phase 3 VS Phase 4 when Task (T) executes as shown in Figure 6.

Figure 5 shows the average voltage for phase 3 compared to phase 4 when task execution in the mixed-criticality system occurs. It can be seen that the decrease of the average voltage for LED, LCD and Loudspeaker in phase 4 compared to phase 3. The decrease of voltage in phase 4 is 0.049323455 for LED, 0.022344457 for LCD and 0.313630818 for Loudspeaker. It can be seen that the advantage of DPM method take place in phase 4 whenever the priority assignation is done at early process. 
Table 5. Different in Voltage Value Measured for Phase 3 and Phase 4

\begin{tabular}{|c|c|c|c|c|c|c|}
\hline \multicolumn{6}{|c|}{ Voltage measured when task execute in MC system (V) } \\
\hline & \multicolumn{3}{|c|}{ Phase 4 } & \multicolumn{3}{c|}{ Phase 3 } \\
\hline No. of samples & LED & LCD & Loudspeaker & LED & LCD & Loudspeaker \\
\hline 0 & 3.184471 & 1.509118 & 0.797737 & 3.183182 & 1.496231 & -0.014165 \\
\hline 0.001 & 3.207668 & 2.357104 & 1.598041 & 3.228288 & 2.333907 & -0.06958 \\
\hline 0.002 & 3.174161 & 2.719238 & 2.152196 & 3.211534 & 2.702485 & -0.083756 \\
\hline 0.003 & 3.148387 & 2.867443 & 2.476957 & 3.192203 & 2.867443 & -0.095355 \\
\hline 0.004 & 3.135499 & 2.930591 & 2.665112 & 3.181894 & 2.944767 & -0.097932 \\
\hline 0.005 & 3.129056 & 2.96152 & 2.769499 & 3.172872 & 2.98214 & -0.099221 \\
\hline 0.006 & 3.126478 & 2.974408 & 2.827492 & 3.17545 & 3.004048 & -0.099221 \\
\hline 0.007 & 3.122612 & 2.980851 & 2.860999 & 3.172872 & 3.010492 & -0.10051 \\
\hline 0.008 & 3.118746 & 2.984717 & 2.879041 & 3.172872 & 3.016936 & -0.097932 \\
\hline 0.009 & 3.122612 & 2.986006 & 2.89064 & 3.174161 & 3.016936 & -0.096644 \\
\hline 0.01 & 3.126478 & 2.989872 & 2.898372 & 3.180605 & 3.015647 & -0.091489 \\
\hline
\end{tabular}

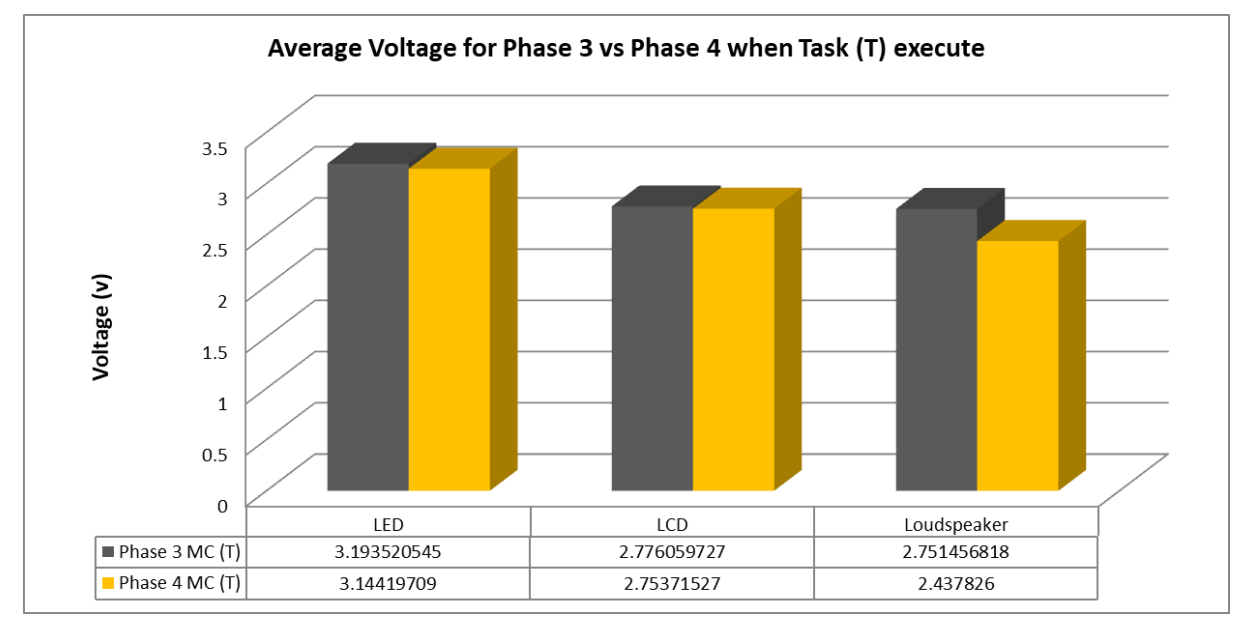

Figure 6. Average voltage for Phase 3 VS Phase 4 when Task (T) executes

\section{CONCLUSION}

The main focus of this project is to fulfil 3 objectives, which are to analyze available energy-saving technique in mixed-criticality system for DPM method, to develop DPM method for mixed-criticality system in microcontroller, and to evaluate the DPM method in preserving energy for mixed-criticality system in microcontroller. Based on the project done using the DPM method to save energy in microcontroller contain mixed-criticality system, it shows that the energy can be saved up to $5 \%$ when task execution occurs for phase 4. Thus, implementation of DPM method can be done to save energy and can help to prolong battery life in the microcontroller.

Improvements that can be done in the future are implementation of DVFS method for mixedcriticality system in microcontroller to see the effectiveness of the method in order to preserve energy, combination of DPM method and DVFS method for mixed-criticality system in microcontroller to save more energy when task execution occurs, increase the complexity of mixed-criticality system to see effectiveness of DPM or DVFS method in order to be used in bigger systems and implementation of the DPM or DVFS method in other microcontroller board such as Arduino or MCU 8051 to see how the method works in different microcontroller environment.

\section{ACKNOWLEDGEMENTS}

The work presented in this paper was supported by the UTM GUP Tier 1 grant, Q.K130000.2538.17H44

\section{REFERENCES}

[1] Bogdanov, L. V., \& Ivanov, R. M. (2012). "Approaches for reducing the power consumption in embedded systems". Annual Journal of Electronics, 1-4.

[2] Ali, I., Seo, J.-h., \& Kim, K. H. (2015). "A Dynamic Power-Aware Scheduling of Mixed-Criticality Real-Time Systems". 2015 IEEE International Conference on Computer and Information Technology; Ubiquitous Computing 
and Communications; Dependable, Autonomic and Secure Computing; Pervasive Intelligence and Computing (pp. 438 - 445). Liverpool, UK: IEEE.

[3] Burns, A., \& Davis, R. I. (December 2017). "A Survey of Research into Mixed Criticality Systems". ACM Transactions on Embedded Computing Systems (Vol. 50, No. 6, Article 82), 1-35.

[4] Ahmed, S. U., Azmi, M. A., \& Badgujar, C. (January 2014). "How to design and test safety critical software systems". International Journal of Advances in Computer Science and Technology (Volume3, No.1), 19-22.

[5] Baruah, S. (April 2018). "Mixed-Criticality Scheduling Theory: Scope, Promise, and Limitations". IEEE Design \& Test (Volume:35, Issue:2), 31-37.

[6] Baruah, S., \& Burns, A. (June 2011). Implementing mixed criticality system in Ada. Ada-Europe'11 Proceedings of the 16th Ada-Europe International conference on Reliable software technologies (pp. 174-188). Edinburgh, UK: Springer

[7] Vestal, S. (December 2007). Preemptive Scheduling of Multi-criticality Systems with Varying Degrees of Execution Time Assurance. 28th IEEE International Real-Time Systems Symposium (RTSS 2007) (pp. 239-243). Tucson, AZ, USA: IEEE

[8] Manna, U., \& N.Banait, S. (2017). "COTS Based Low Energy Multiprocessor Embedded System". International Journal of Electrical and Electronics Engineers (Vol No.9, Issue No.01), 1097-1102.

[9] Zimmer, M., Broman, D., Shaver, C., \& Lee, E. A. (2014). "Flexpret: A Processor Platform for Mixed-Criticality Systems". 2014 IEEE 19th Real-Time and Embedded Technology and Applications Symposium (RTAS) (pp. 101 110). Berlin, Germany: IEEE.

[10] Li, H., \& Baruah, S. (2012). "Global Mixed-Criticality Scheduling on Multiprocessors". 24th Euromicro Conference on Real-Time Systems, ECRTS 2012 (pp. 166-175). Pisa, Italy: Proceedings of the 24th Euromicro Conference on Real-Time Systems, ECRTS 2012.

[11] Benini, L., Bogliolo, A., \& Micheli, G. D. (June 2000). "A Survey of Design Techniques for System-Level Dynamic Power Management". IEEE Transactions on Very Large Scale Integration (VLSI) Systems ( Vol: 8 , Issue: 3), 299 316.

[12] Suleiman, D. R., Ibrahim, M. A., \& Hamarash, I. I. (December 2005). "Dynamic Voltage Frequency Scaling (DVFS) for Microprocessors Power and Energy Reduction". CiteSeerX, 1-5.

[13] Pering, T., Burd, T., \& Brodersen, R. (July 2016). "Dynamic Voltage Scaling and the Design of a Low-Power Microprocessor System". CiteSeerX, 1-6.

[14] Li, H., \& Baruah, S. (2010). "An Algorithm for Scheduling Certifiable Mixed-Criticality Sporadic Task Systems". 201031 st IEEE Real-Time Systems Symposium (pp. 183 - 192). San Diego, CA, USA: IEEE.

[15] Rathod, M. P., \& Gangane, P. J. (June 2016 ). "Multi Controller Based High Efficient System with DVFS and DPM Control". International Journal of Innovative Research in Science, Engineering and Technology (Vol. 5, Issue 6), 10996-11000.

[16] Salehi, M., \& Ejlali, A. (2015). "A Hardware Platform for Evaluating Low-Energy Multiprocessor Embedded Systems Based on COTS Devices". IEEE Transactions on Industrial Electronics (Vol. 62, No. 2), 1262-1269.

\section{BIOGRAPHIES OF AUTHORS}

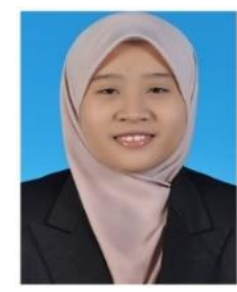

Siti Nurhafizza binti Maidin work at Infinecs Systems Sdn. Bhd. as design engineer. She obtained her B.Eng. in Computer Engineering in 2016 from Universiti Teknologi MARA and Master of Science, majoring in Computer System Engineering in 2018 from Universiti Teknologi Malaysia. She is Registered Graduate Engineer with the Board of Engineers Malaysia (BEM). She actively involved in extra curriculum such as IEEE UiTM Student Branch secretary, board member of college community, facilitator for Arduino program and netball member of Electrical faculty in UiTM. She has been actively involved in research related to wireless networking, Internet of Things (IoT), embedded systems and system engineering during her master study.

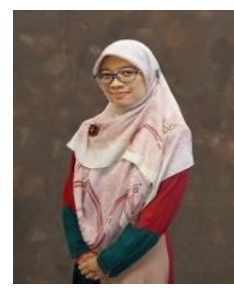

Dr. Noor Azurati binti Ahmad serves as an Associate Professor and the Deputy Director of Innovation and Commercialization Center in Universiti Teknologi Malaysia Kuala Lumpur. She obtained her B.Eng. in Computer Engineering in 2001 and Master of Electrical Engineering in 2006 from Universiti Teknologi Malaysia. She graduated with a PhD in Embedded Systems from University of Leicester in 2013. She is a Certified Tester Foundation Level (CTFL) under Malaysian Software Testing Board (MSTB) and Certified Professional for Requirements Engineering (CPRE) under International Requirements Engineering Board (IREB). She has also served as an engineer in Sapura Secured Technologies Sdn. Bhd under the Network Centric Operations (NCO) project. She involved in SIRIM qualification test for Sapura's military products. She is a member of Institute Electrical Electronic Engineer (IEEE), IEEE Computer Society and Registered Graduate Engineer with the Board of Engineers Malaysia (BEM). She has been actively involved in research related to design, software architecture and testing for embedded real-time systems and mobile and pervasive computing. 




Kamilia Kamardin received her B.Eng. Electronic (Communications) from the University of Sheffield, U.K., in 2004 and obtained her M.Sc. in Information Technology (Data Communications and Networking) from Universiti Teknologi Mara (UiTM), Malaysia in 2007. She received her Ph.D. in Electrical Engineering (Communications) from Universiti Teknologi Malaysia (UTM), Malaysia in 2014. She spent 3 months at University of Bimingham, U.K., as a visiting Ph.D. student. She has previously served as a senior assistant researcher at TM Research $\&$ Development, Malaysia for 3 years. Currently she serves as senior lecturer at Department of Electronic Systems Engineering, Malaysia-Japan International Institute of Technology (MJIIT), Universiti Teknologi Malaysia (UTM), Kuala Lumpur. She is a member of Communication Systems and Network (CSN) iKohza. She is also an associate member of Wireless Communication Centre (WCC) and Cyber Physical Systems (CPS) Research Group at UTM. She is a Professional Engineer under Board of Engineers Malaysia (BEM) and Professional Technologies under Malaysia Board of Technologies (MBOT). Also, she is a Senior Member of Institute of Electrical and Electronics Engineers (IEEE) and currently is serving as the Treasurer of IEEE Malaysia Antennas and Propagation, Microwave Theory \& Techniques and Electromagnetic Compatibility (AP/MTT/EMC) Joint Chapter. Her research interests include antennas and propagation, wireless communication, wearable communication, body centric communication, metamaterials and Internet of Things (IoT).

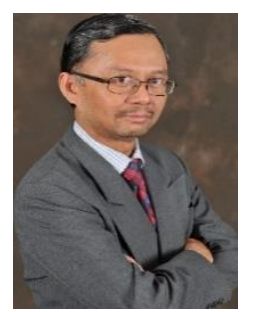

Shamsul Sahibuddin is a Professor of Software Engineering at Universiti Teknologi Malaysia (UTM). He is currently the Dean of Advanced Informatics School in UTM. He graduated from Aston University, United Kingdom with Ph.D. in Computer Science. He also holds masters and bachelor degree in computer science. His research interest is in software and system engineering, software quality, software process improvement, secure software development, requirement engineering, and information technology service management. He has twenty nine years experiences in software development and lecturing. He also is part of the Asia Pacific Software Engineering Conference Steering Committee since 2009. He has been the Chairman of the Technical Committee 11 on Software Engineering Standard at SIRIM, Malaysia since 2000.



Sharizal Fadlie bin Sabri work as engineer at Pusat Angkasa Negara. He obtained bachelor of Computer Science (Artificial Intelligence) in 2004 at University Malaya and holds Master of Science (Computer System Engineering) in 2016 in Universiti Teknologi Malaysia. He is currently persuade his $\mathrm{PhD}$ at Universiti Teknologi Malaysia, research in Dynamic Voltage Frequency Scaling (DVFS). He awarded as excellent service award in 2011 by National Space Agency, Ministry of Science, Technology and Innovation. Some of the paper had been published by him are Energy performance evaluation for dynamic frequency scaling on rate monotonic and earliest deadline first scheduling algorithm, Implementation of Cryptography Algorithms in Scadakratos Application, Design and development of Motorized Adjustable Vertical Platform (MAVeP) for satellite test facility and Development of Computer Designated Mode Gateway for Ground Station Controller Software. 